\title{
Validation of the custo screen 400 ambulatory blood pressure-monitoring device according to the European Society of Hypertension International Protocol revision 2010
}

\author{
This article was published in the following Dove Press journal: \\ Vascular Health and Risk Management \\ 13 May 2014 \\ Number of times this article has been viewed
}

\author{
Peter Bramlage' \\ Cornelia Deutsch' \\ Ralf Krüger' \\ Andreas Wolf ${ }^{2}$ \\ Peter Müller ${ }^{2}$ \\ Thomas Zwingers ${ }^{1,4}$ \\ Beate Beime' \\ Thomas Mengden ${ }^{3}$ \\ 'Institut für Pharmakologie und \\ Präventive Medizin, Cloppenburg, \\ 2Müller and Sebastiani, Ottobrunn, \\ ${ }^{3}$ Kerckhoff-Klinik, Bad Nauheim, \\ ${ }^{4}$ Estimate, Augsburg, Germany
}

Correspondence: Peter Bramlage Institut für Pharmakologie und Präventive Medizin, Bahnhofstrasse 20, 4966I Cloppenburg, Germany Tel +49 447| 850 333 I

Fax +49 447I 8503332

Email peter.bramlage@ippmed.de
Objective: The aim of the present study was to validate the custo screen 400 ambulatory blood pressure-monitoring (ABPM) device according to the 2010 International Protocol revision of the European Society of Hypertension (ESH-IP). The device can be used for ABPM for up to 72 hours.

Materials and methods: Systolic and diastolic blood pressure (SBP and DBP, respectively) were sequentially measured in 33 adult subjects (13 males and 20 females) and compared with a standard mercury sphygmomanometer (two observers). A total of 99 comparison pairs were obtained.

Results: The custo screen 400 met the requirements of parts 1 and 2 of the ESH-IP revision 2010. The mean difference between the device and reference sphygmomanometer readings was $-0.5 \pm 4.5 \mathrm{mmHg}$ for SBP and $-0.1 \pm 3.3 \mathrm{mmHg}$ for DBP. All but one measurement were within the absolute difference of $10 \mathrm{mmHg}$ between the device and the observers for SBP and DBP. The number of absolute differences between the device and the observers within a range of $5 \mathrm{mmHg}$ was 84 of 99 readings for SBP, and 93 of 99 readings for DBP.

Conclusion: The custo screen 400 ABPM device met the requirements of the 2010 ESH-IP revision, and hence can be recommended for ABPM in adults. To our knowledge, the custo screen 400 is the first device to pass the revised ESH-IP 2010.

Keywords: validation, ambulatory blood pressure monitoring, ESH

\section{Introduction}

The importance of ambulatory blood pressure monitoring (ABPM) is emphasized in the 2013 European Society of Hypertension (ESH)/European Society of Cardiology (ESC) guidelines for the management of arterial hypertension. ${ }^{1}$ Furthermore, the ESH recently published a position paper on $\mathrm{ABPM},{ }^{2}$ reinforcing the need for proper validation of BP-measuring devices according to the revised ESH International Protocol (IP) 2010 criteria. $^{3}$ Surprisingly, no reports on ABPM devices that have successfully passed the 2010 version of the ESH-IP have been published to date. ${ }^{4}$

The custo screen 400 (custo med, Ottobrunn, Germany) is a newly developed ABPM device for 24-hour BP measurement. An important feature is the option to use it as a Holter ABPM recorder for the synchronous recording of ABPM and Holter electrocardiograms (ECGs). The custo screen 300 is identical to the custo screen 400, but without an ECG-recording facility. Both systems are intended for use by trained specialist staff or physicians in clinics and offices. The aim of the present study was 
to evaluate the accuracy of BP measurements obtained with the custo screen 400, as assessed according to the criteria of the ESH-IP revision $2010 .^{3}$

\section{Device details}

The custo screen 400 is an ABPM device that is used for recording and evaluating a subject's BP (Figure 1). As a special feature, it can be used in combination with custo guard 3 (a three-channel ECG transmitter) for synchronized ABPM and Holter ECG recordings. The custo screen 400 is an oscillometric measuring device with automatic zero balancing. The maximum recording time is 72 hours for ABPM recordings alone and 24 hours for synchronous Holter ABPM recordings. The cuff pressure ranges from 0 to $300 \mathrm{mmHg}$. The standard adult cuff can be used for arm circumferences of 24-32 cm. Further cuff sizes are available for arm circumferences of 20-24 cm (small), 32-40 cm (XL), and 38-50 cm (XXL).

To initiate ABPM, the custo screen infrared interface must be connected to the computer and be ready for operation. After selecting a subject, different parameters have to be set, including measuring intervals (standard or customized), time for day and night phases or additional phases, the option for repeated measurements, signal before measurement (beep), display results, and print diary. The display of the device indicates systolic and diastolic BP (SBP and DBP, respectively) values together with heart rate and an error code in case of failure. When the measurements are complete, data are downloaded via an infrared interface. The quality of the recording, including invalid measurements and their underlying causes, can be checked.

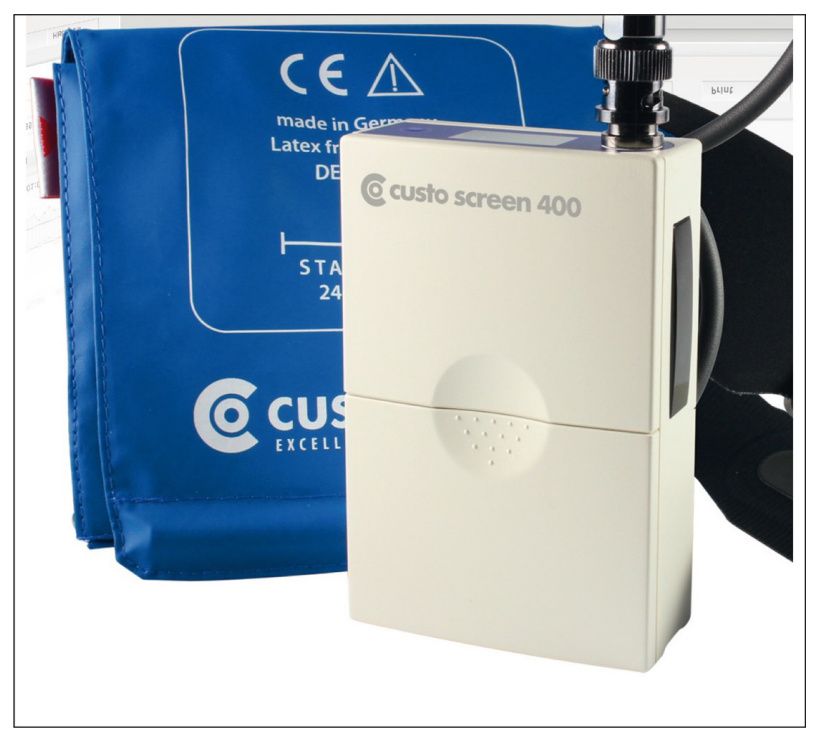

Figure I Custo screen 400 test device.

\section{Materials and methods}

\section{Familiarization}

Prior to the validation procedure, the validation team participated in a familiarization session, and a series of BP measurements were taken using the test device. The custo screen 400 was determined to function correctly, with no abnormalities identified.

\section{Recruitment}

Subjects were recruited at the Institut für Pharmakologie und Präventive Medizin in Cloppenburg, Germany. Subjects were not eligible for the study if they had arrhythmia, Korotkoff sounds of poor quality, or an arm circumference outside the cuff range. A total of 37 subjects aged at least 25 years were screened, and 33 subjects were enrolled. Written informed consent was obtained from each of the subjects.

\section{Procedure}

The test strictly followed the recommendations of the ESH-IP revision 2010 for the validation of ABPM devices in adults. ${ }^{3}$ Measurements were performed by two experienced, trained observers and a supervisor. The observers were instructed in the use of the custo screen 400.

Each subject was seen individually in a quiet, temperature-controlled room. Measurements were performed in a sitting position with the arm supported at heart level after resting for 10-15 minutes, in accordance with the ESH-IP guidelines. The upper-arm circumference was measured to ensure that the cuff was of adequate size, but selection of the subjects was not made on the basis of their arm circumference. For reference measurements, standard BP auscultation with a mercury sphygmomanometer (ERKAmeter 3000; ERKA, Bad Tölz, Germany) and an appropriate reference cuff was used. The standard sphygmomanometer was checked and calibrated using a KAL 100 (Halstrup-Walcher, Kirchzarten, Germany). Reference measurements were performed by two experienced and trained observers, who determined the auscultation readings simultaneously in a blinded manner with a dual-earpiece teaching stethoscope.

Nine BP measurements were taken with the reference standard sphygmomanometer and the custo screen 400 for each subject. Baseline BP values were measured by the observers (BPA), and used to categorize the subjects within the following BP ranges: 90-129, 130-160, and 161-180 mmHg for SBP, and 40-79, 80-100, and 101-130 mmHg for DBP. After measurement of device-detection BP (BPB), seven alternate measurements with the standard sphygmomanometer 
(BP1, BP3, BP5, and BP7) and the test device (BP2, BP4, and BP6) were performed for analysis.

\section{Statistical analysis}

Analysis was performed using SAS version 9.3 (SAS Institute, Cary, NC, USA) and Microsoft Excel 2010 (Microsoft, Redmond, WA, USA). SBP and DBP were analyzed separately. The mean of each pair of observer measurements was calculated as a reference value. The differences between the mean observer value and the device values were calculated according to ESH-IP 2010 and were displayed in Bland-Altman plots against the difference between the two values.

\section{Results}

\section{Subjects}

A total of 37 subjects were screened, and 33 subjects were enrolled (Table 1). Four subjects were excluded: one had a $\mathrm{BP}$ range for a group that was fully enrolled, one had been assigned to the wrong BP range, and two were disregarded to achieve the proper BP distribution with respect to DBPrange criteria.

Table I Screening and recruitment details

\begin{tabular}{|c|c|c|c|}
\hline \multicolumn{4}{|c|}{ Screening and recruitment $(n=37)$} \\
\hline \multicolumn{2}{|l|}{ Total screened } & \multicolumn{2}{|l|}{37} \\
\hline \multicolumn{2}{|l|}{ Total excluded } & \multicolumn{2}{|l|}{4} \\
\hline Ranges complete & 0 & & \\
\hline Range adjustment & 2 & & \\
\hline Arrhythmias & 0 & & \\
\hline Device failure & 0 & & \\
\hline Poor-quality sounds & 0 & & \\
\hline Cuff size unavailable & 0 & & \\
\hline Observer disagreement & 0 & & \\
\hline Distribution & 2 & & \\
\hline Other reasons & 0 & & \\
\hline \multirow[t]{2}{*}{ Total recruited } & & \multicolumn{2}{|l|}{33} \\
\hline & $\mathrm{mmHg}$ & All & On $\mathbf{R x}$ \\
\hline \multicolumn{4}{|c|}{ Recruitment ranges $(n=33)$} \\
\hline \multicolumn{4}{|l|}{ SBP } \\
\hline \multirow{2}{*}{ Low } & $<90$ & 0 & \multirow{2}{*}{4} \\
\hline & $90-129$ & 11 & \\
\hline Medium & $130-160$ & 10 & 5 \\
\hline \multirow{2}{*}{ High } & $161-180$ & 11 & \multirow{2}{*}{8} \\
\hline & $>180$ & I & \\
\hline \multicolumn{4}{|l|}{ DBP } \\
\hline \multirow{2}{*}{ Low } & $<40$ & 0 & \multirow{2}{*}{5} \\
\hline & $40-79$ & 11 & \\
\hline Medium & $80-100$ & II & 5 \\
\hline \multirow{2}{*}{ High } & $101-130$ & II & \multirow{2}{*}{7} \\
\hline & $>130$ & 0 & \\
\hline
\end{tabular}

Abbreviations: SBP, systolic blood pressure; DBP, diastolic blood pressure; $\mathrm{Rx}$, antihypertensive drug prescription.
Table 2 Subject characteristics at baseline

\begin{tabular}{lllll}
\hline & Mean & SD & Min & Max \\
\hline Sex & & & & \\
$\quad$ Male:female & $13: 20$ & & & \\
$\quad$ Age (years) & 57.4 & 13 & 25 & 87 \\
$\quad$ Arm circumference $(\mathrm{cm})$ & 28.9 & 2.9 & 24 & 36 \\
Cuff size for test device & & & & \\
$\quad$ Standard $(24-32 \mathrm{~cm}), \mathrm{n}(\%)$ & $31(93.9)$ & & & \\
$\quad$ Large $(32-40 \mathrm{~cm}), \mathrm{n}(\%)$ & $2(6.1)$ & & & \\
SBP $(\mathrm{mmHg})$ & 143.9 & 28.1 & 106 & 218 \\
DBP $(\mathrm{mmHg})$ & 87.9 & 17.9 & 49 & 123 \\
\hline
\end{tabular}

Abbreviations: SBP, systolic blood pressure; DBP, diastolic blood pressure; $\mathrm{SD}$, standard deviation.

The remaining 33 subjects ( 13 males and 20 females) had a mean age of 57.4 \pm 13 years (range $25-87$ years) (Table 2). Mean arm circumference was $28.9 \pm 2.9 \mathrm{~cm}$ (range 24-36 cm). With the exception of two subjects who required a larger cuff size, all subjects had an arm circumference that was suitable for the standard cuff. At baseline, mean SBP was 143.9 $\pm 28.1 \mathrm{mmHg}$ (range 106-218 mmHg), and mean DBP was $87.9 \pm 17.9 \mathrm{mmHg}$ (range $49-123 \mathrm{mmHg}$ ). For each subject, three valid BP measurements were taken with the custo screen 400, resulting in 99 valid measurements for analysis.

\section{Blood pressure measurements}

Table 3 demonstrates that all mean observer test measurements were within the range of 25-43, as specified by ESH-IP 2010. In addition, the maximum difference between categories for SBP and DBP was below the specified limit of 19 . The range of observer measurements was 100-220 $\mathrm{mmHg}$ for SBP, and 49-123 mmHg for DBP. Overall mean observer differences were $0.4 \pm 1.4$ and $-0.4 \pm 1.5 \mathrm{mmHg}$ for SBP and DBP, respectively (Table 4).

The mean difference ( \pm standard deviation) between measurements obtained with the custo screen 400 and the reference sphygmomanometer was $-0.5 \pm 4.5 \mathrm{mmHg}$ for SBP and $-0.1 \pm 3.3 \mathrm{mmHg}$ for DBP (Table 5). Based on the ESH-IP 2010 criteria, the device met the pass requirements for SBP and DBP in parts 1 and 2.

Table 3 Observer measurements for subjects within each BP range

\begin{tabular}{lll}
\hline & SBP $(\mathbf{m m H g})$ & DBP $(\mathbf{m m H g})$ \\
\hline Overall range (low-high) & $100-220$ & $49-123$ \\
Low (SBP $<$ I30, DBP $<80)$ & 43 & 40 \\
Medium (SBP 130-160, DBP 80-100) & 30 & 34 \\
High (SBP > 160, DBP $>$ 100) & 26 & 25 \\
Maximum difference & 17 & 15 \\
\hline
\end{tabular}

Abbreviations: SBP, systolic blood pressure; DBP, diastolic blood pressure. 
Table 4 Observer differences

\begin{tabular}{llll}
\hline & $\begin{array}{l}\text { SBP } \\
(\mathbf{m m H g})\end{array}$ & $\begin{array}{l}\text { DBP } \\
(\mathbf{m m H g})\end{array}$ & $\begin{array}{l}\text { Repeated } \\
\text { measurements }\end{array}$ \\
\hline Observer 2 - Observer I & & \\
Range & -2 to 4 & -4 to 4 & NA \\
Mean (SD) & $0.4(\mathrm{I} .4)$ & $-0.4(\mathrm{I}) 5)$ & NA \\
\hline
\end{tabular}

Abbreviations: SBP, systolic blood pressure; DBP, diastolic blood pressure; $\mathrm{SD}$, standard deviation; NA, not applicable.

Bland-Altman plots of the differences between BP measurements obtained with the custo screen 400 and the standard reference sphygmomanometer (Figures 2 and 3) demonstrated an equal distribution of BP differences around $0 \mathrm{mmHg}$, with only one outlier beyond $10 \mathrm{mmHg}$. The majority of values were within a range of $5 \mathrm{mmHg}$ for both SBP and DBP.

\section{Discussion}

To our knowledge, the custo screen 400 device is the first to pass the ESH-IP 2010 revision. ${ }^{3}$ A PubMed search (http://www. pubmed.org), which was conducted on February 28, 2014 using the search string "ESH International Protocol 2010", resulted in 20 publications on the validation of BP-measuring devices. Of those, 13 reported the outcomes of validation studies for a total of 19 devices; however, none of these publications reported the outcomes of a device intended for ABPM use. Using the dabl ${ }^{\circledR}$ Educational Trust website (http://www.dableducational.org) on February 28, 2014, a search for ABPM devices undergoing validation according to the ESH-IP resulted in 21 studies in which 37 devices were tested. Among these studies, 21 devices were "recommended", seven were considered to be "questionable", and nine were "not recommended". All these devices were validated according to ESH-IP 2002, but not ESH-IP 2010. As outlined in a recent overview comparing 2002 with the 2010 version of ESH-IP, Stergiou et $\mathrm{al}^{4}$ concluded that the
2010 criteria were stricter, especially with respect to the passlevel criteria, ${ }^{3}$ and would noticeably increase the failure rate of devices being validated.

A recent systematic review ${ }^{5}$ on the accuracy of ABPMs confirmed the search results outlined earlier of no published validations as to ESH-IP 2010, ${ }^{3}$ and also considered further validation protocols, such as those of the British Hypertension Society (BHS), ${ }^{6}$ the Association for the Advancement of Medical Instrumentation (AAMI), ${ }^{7}$ and the International Organization for Standardization. ${ }^{8}$ They identified a total of 38 studies, of which 28 assessed a monitor in the general population. Of these, protocols were passed in 24 of 28 studies, but 12 of 24 (50\%) found a difference of at least $5 \mathrm{mmHg}$ SBP between the test device and the reference standard for $30 \%$ or more of the readings.

The authors also assessed the effect of the introduction of ESH-IP 2010 on ABPM-validation studies in a retrospective comparison of included studies, and estimated the proportion passing or failing each protocol, based on testing the device accuracy per measurement alone. ${ }^{5}$ The analyses demonstrated that eight studies - of which four passed original validation using ESH-IP1 (2002) $)^{9-12}$ and four using the BHS protocol $^{13-16}$ - would have passed IP1 but failed the more rigorous criteria of IP2 (2010). Overall, the pass rate in studies using the ESH-IP protocol would have dropped from $83 \%$ (ten of 12 ) to $50 \%$ (six of 12) if the IP2 criteria had been used instead, a finding that has been previously reported. ${ }^{4} \mathrm{~A}$ further five studies ${ }^{17-21}$ that passed monitors using the BHS criteria would have failed using either ESH-IP grading.

Putting our results into perspective with the results obtained by evaluating clinical studies as to the ESH-IP2 criteria, we obtained excellent results for both SBP (84 of 99 or $85 \%$ were $\leq 5 \mathrm{mmHg}$ ) and DBP readings ( 93 of 99 or

Table 5 Validation results

\begin{tabular}{|c|c|c|c|c|c|c|}
\hline Part I & $\leq 5 \mathrm{mmHg}$ & $\leq 10 \mathrm{mmHg}$ & $\leq 15 \mathrm{mmHg}$ & Grade I & Mean $(\mathrm{mmHg})$ & $\mathrm{SD}(\mathrm{mmHg})$ \\
\hline \multicolumn{7}{|l|}{ Pass requirements } \\
\hline Two of & 73 & 87 & 96 & & & \\
\hline All of & 65 & 81 & 93 & & & \\
\hline \multicolumn{7}{|l|}{ Achieved } \\
\hline SBP & 84 & 98 & 99 & Passed & -0.5 & 4.5 \\
\hline DBP & 93 & 99 & 99 & Passed & -0.1 & 3.3 \\
\hline Part 2 & $2 / 3 \leq 5 \mathrm{mmHg}$ & $0 / 3 \leq 5 \mathrm{mmHg}$ & & Grade 2 & & Grade 3 \\
\hline Pass requirements & $\geq 24$ & $\leq 3$ & & & & \\
\hline \multicolumn{7}{|l|}{ Achieved } \\
\hline SBP & 30 & 0 & & Passed & & \\
\hline DBP & 32 & I & & Passed & & \\
\hline \multirow[t]{2}{*}{ Part 3} & & & & & & Result \\
\hline & & & & & & Passed \\
\hline
\end{tabular}

Abbreviations: SBP, systolic blood pressure; DBP, diastolic blood pressure; SD, standard deviation. 


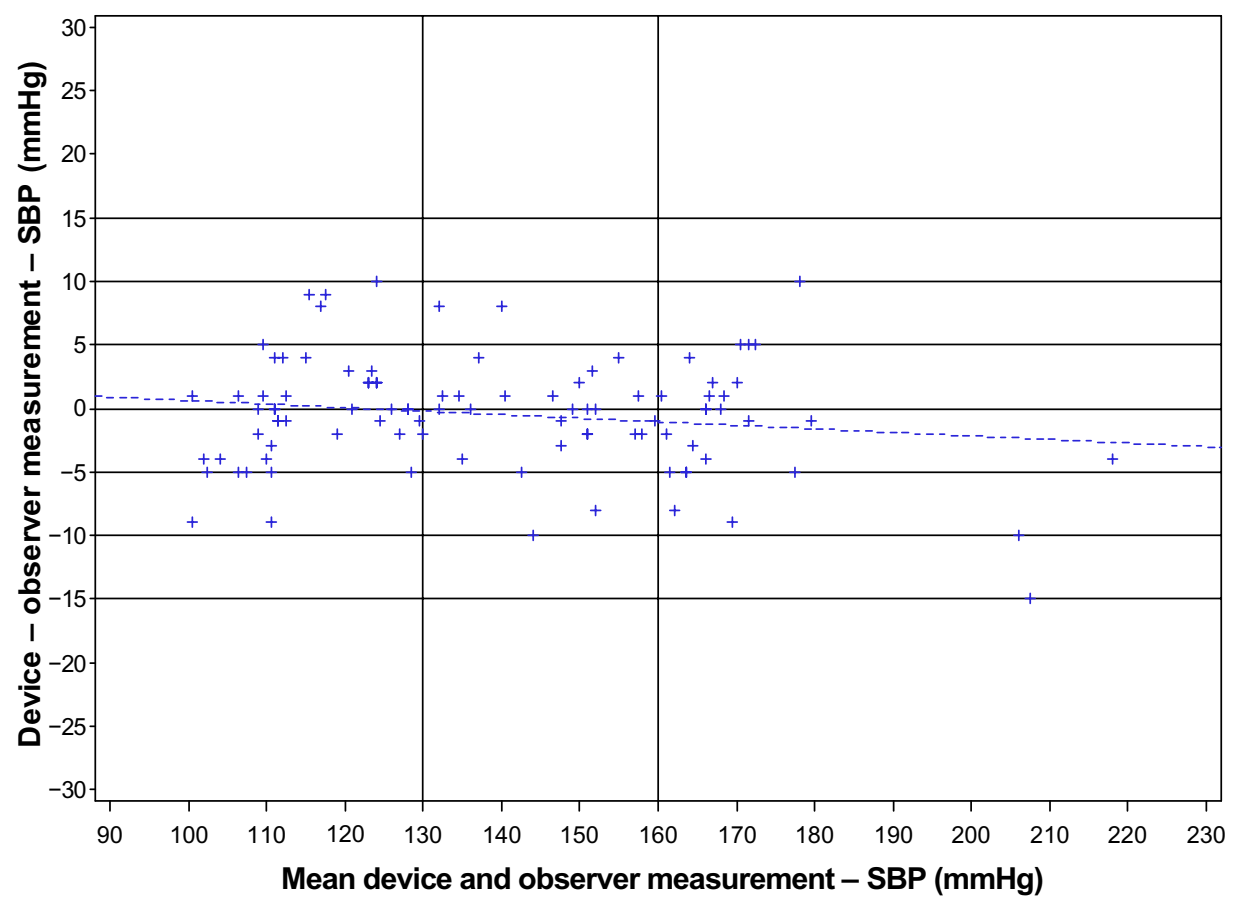

Figure 2 Bland-Altman plots demonstrating the systolic blood pressure (SBP) differences between the custo screen 400 device and reference sphygmomanometer and the average of device and observer pressure values.

$94 \% \leq 5 \mathrm{mmHg}$ ). Comparable results were only obtained by six of 28 previous studies in the general population using IP1, AAMI or BHS, being evaluated post hoc using the IP2 criteria, ${ }^{22-27}$ with comparatively better results for the SBP than the DBP reading.
Noteworthy are limitations of current protocols and the low adherence of investigators to these. This has been previously acknowledged, ${ }^{5}$ with pledges for simple and straightforward instructions, such as in the most recent ESH-IP2. On the other hand, these protocols do not cover

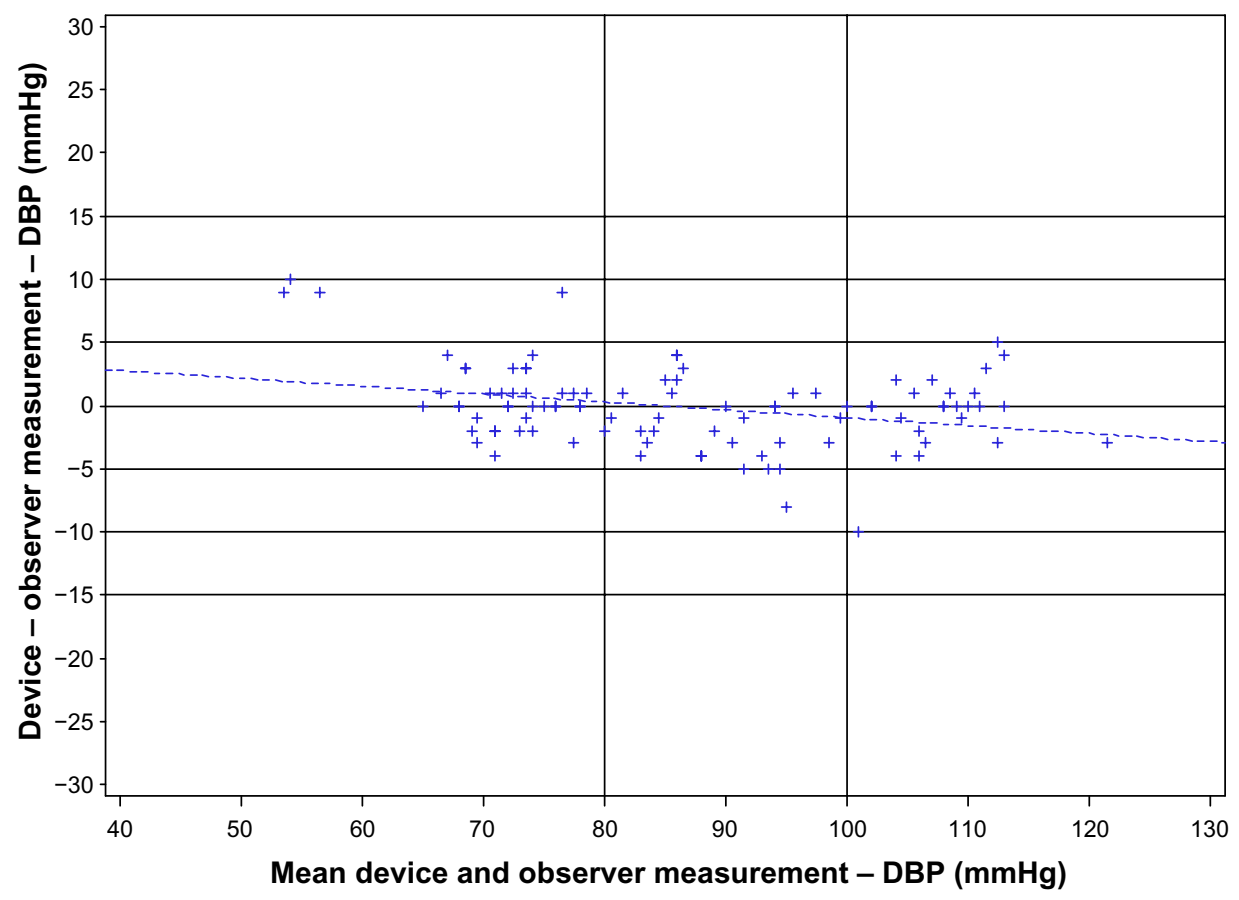

Figure 3 Bland-Altman plots demonstrating the diastolic blood pressure (DBP) differences between the custo screen 400 device and reference sphygmomanometer and the average of device and observer pressure values. 
typical clinical situations, such as patients lying during sleep. This has been fostered by the BHS 1993 protocol revision, where a second part of $\mathrm{ABPM}$ validation is recommended during exercise and different postures. ${ }^{6}$ Current protocols therefore need to balance the request for more elaborate validations on the one hand, and the obvious difficulties in adhering to even simpler protocols, as is evident from prior research. As Hodgkinson et al concluded from their analyses, ESH-IP2 appears without compromise with respect to the integrity of the validation procedure, though simpler than prior protocol versions. ${ }^{5}$

\section{Perspectives}

A new feature of the custo screen 400 is the possibility to use it in combination with a Holter ECG device for synchronous recording of ABPM and Holter ECG for up to 24 hours. This may be an advantage for a considerable subgroup of patients with suspected rhythm disorders, in whom BP measurements tend to be inaccurate or lead to errors of the test device. Further studies are needed to explore fully the potential of this device combination.

\section{Conclusion}

The custo screen 400 ABPM device passed the ESH-IP 2010 revision requirements, and hence can be recommended for ABPM in adults. To our knowledge, the custo screen 400 device is the first to pass the revised ESH-IP 2010.

\section{Acknowledgment}

Custo med GmbH, Ottobrunn, Germany provided funding for this validation study.

\section{Disclosure}

Andreas Wolf is an employee and Peter Müller a shareholder of Müller and Sebastiani and custo med GmbH, Germany. Thomas Mengden is a consultant for custo med. The remaining authors disclose having performed validation studies for a number of companies producing BP monitors, for which the institute was paid, and report no other conflicts of interest in this work.

\section{References}

1. Mancia G, Fagard R, Narkiewicz K, et al. 2013 ESH/ESC Guidelines for the management of arterial hypertension: the Task Force for the management of arterial hypertension of the European Society of Hypertension (ESH) and of the European Society of Cardiology (ESC). J Hypertens. 2013;31:1281-1357.

2. O'Brien E, Parati G, Stergiou G, et al. European Society of Hypertension position paper on ambulatory blood pressure monitoring. J Hypertens. 2013;31:1731-1768.
3. O'Brien E, Atkins N, Stergiou G, et al. European Society of Hypertension International Protocol revision 2010 for the validation of blood pressure measuring devices in adults. Blood Press Monit. 2010;15:23-38.

4. Stergiou GS, Karpettas N, Atkins N, O'Brien E. Impact of applying the more stringent validation criteria of the revised European Society of Hypertension International Protocol 2010 on earlier validation studies. Blood Press Monit. 2011;16:67-73.

5. Hodgkinson JA, Sheppard JP, Heneghan C, et al. Accuracy of ambulatory blood pressure monitors: a systematic review of validation studies. J Hypertens. 2013;31:239-250.

6. O'Brien E, Petrie J, Littler W, et al. An outline of the revised British Hypertension Society protocol for the evaluation of blood pressure measuring devices. J Hypertens. 1993;11:677-679.

7. Association for the Advancement of Medical Instrumentation. American National Standard: Electronic or Automated Sphygmomanometer. Arlington (VA): AAMI; 1993.

8. International Organization for Standardization. ISO/OCE 81060-2:2013. Non-invasive sphygmomanometers - Part 2: Clinical validation of automated measurement type. Available from: http://www.iso.org/iso/ iso_catalogue/catalogue_tc/catalogue_detail.htm?csnumber=57977. Accessed April 10, 2014.

9. Denchev SV, Simova II, Matveev MG. Evaluation of the Schiller BR-102 plus noninvasive ambulatory blood pressure monitor according to the International Protocol introduced by the Working Group on Blood Pressure Monitoring of the European Society of Hypertension. Blood Press Monit. 2007;12:329-333.

10. Jones SC, Bilous M, Winship S, Finn P, Goodwin J. Validation of the OSCAR 2 oscillometric 24-hour ambulatory blood pressure monitor according to the International Protocol for the validation of blood pressure measuring devices. Blood Press Monit. 2004;9:219-223.

11. Ragazzo F, Saladini F, Palatini P. Validation of the Microlife WatchBP O3 device for clinic, home, and ambulatory blood pressure measurement, according to the International Protocol. Blood Press Monit. 2010;15:59-62.

12. Octavio JA, Leal N, Contreras J, et al. Accuracy of Jotatec-P24B device for teletransmitted ambulatory blood pressure monitoring. J Hypertens. 2008;26 Suppl 1:S493.

13. Alpert BS. Validation of the Tiba Medical Ambulo 2400 ambulatory blood pressure monitor to the ISO Standard and BHS protocol. Blood Press Monit. 2010;15:275-277.

14. de Gaudemaris R, White A, Pascal I, Siche JP, Baguet JP, Mallion JM. [Evaluation of the ambulatory blood pressure monitor SAVE 33 model 2]. Arch Mal Coeur Vaiss. 1999;92:1133-1137. [French].

15. Goodwin J, Bilous M, Winship S, Finn P, Jones SC. Validation of the Oscar 2 oscillometric 24-h ambulatory blood pressure monitor according to the British Hypertension Society protocol. Blood Press Monit. 2007;12:113-117.

16. Wei W, Tolle M, Zidek W, van der Giet M. Validation of the mobil-OGraph: 24 h-blood pressure measurement device. Blood Press Monit. 2010;15:225-228.

17. Barna I, Keszei A, Dunai A. Evaluation of Meditech ABPM-04 ambulatory blood pressure measuring device according to the British Hypertension Society protocol. Blood Press Monit. 1998;3:363-368.

18. Elvan-Taspinar A, Uiterkamp LA, Sikkema JM, et al. Validation and use of the Finometer for blood pressure measurement in normal, hypertensive and pre-eclamptic pregnancy. J Hypertens. 2003;21: 2053-2060.

19. Franx A, Van Der Post JA, van Montfrans GA, Bruinse HW. Comparison of an auscultatory versus an oscillometric ambulatory blood pressure monitor in normotensive, hypertensive, and pre-eclamptic pregnancy. Hypertens Pregnancy. 1997;16:187-202.

20. O'Brien E, Mee F, Atkins N, Halligan A, O'Malley K. Accuracy of the SpaceLabs 90207 ambulatory blood pressure measuring system in normotensive pregnant women determined by the British Hypertension Society protocol. J Hypertens. 1993;11:S282-S283. 
21. Jones CR, Taylor K, Chowienczyk P, Poston L, Shennan AH. A validation of the Mobil O Graph (version 12) ambulatory blood pressure monitor. Blood Press Monit. 2000;5:233-238.

22. Czarkowski M, Mikulska M, Baran A, Zebrowski M, Rózanowski K. Accuracy of SpaceLabs 90207 is altered by venous blood redistribution. Blood Press. 2003;12:334-339.

23. Nemeth Z, Moczar K, Deak G. Evaluation of the Tensioday ambulatory blood pressure monitor according to the protocols of the British Hypertension Society and the Association for the Advancement of Medical Instrumentation. Blood Press Monit. 2002;7:191-197.

24. Palatini P, Frigo G, Bertolo O, Roman E, Da Corta R, Winnicki M. Validation of the A\&D TM-2430 device for ambulatory blood pressure monitoring and evaluation of performance according to subjects' characteristics. Blood Press Monit. 1998;3:255-260.
25. Langewitz W, Tanner S. Validation of the PhysioQuant blood pressure measuring monitor in adults. Blood Press Monit. 2009;14: 220-221.

26. Pang TC, Brown MA. Accuracy of ambulatory blood pressure monitors in routine clinical practice. Am J Hypertens. 2006;19:801-809.

27. Haensel A, Utech K, Langewitz W. Validation of TONOPORT V bloodpressure measuring monitor in adults. J Hum Hypertens. 2005;19: 745-749.

\section{Publish your work in this journal}

Vascular Health and Risk Management is an international, peerreviewed journal of therapeutics and risk management, focusing on concise rapid reporting of clinical studies on the processes involved in the maintenance of vascular health; the monitoring, prevention and treatment of vascular disease and its sequelae; and the involvement of metabolic disorders, particularly diabetes. This journal is indexed on PubMed Central and MedLine. The manuscript management system is completely online and includes a very quick and fair peer-review system, which is all easy to use. Visit http://www.dovepress.com/ testimonials.php to read real quotes from published authors.

Submit your manuscript here: http://www.dovepress.com/vascular-health-and-risk-management-journal 